\title{
Multi-determinant X-ray restrained wavefunction approaches
}

\author{
A. Genoni \\ Université de Lorraine \& CNRS, Laboratoire de Physique et Chimie Théoriques (LPCT), UMR CNRS 7019, \\ 1 Boulevard Arago, F-57078 Metz, France, \\ Alessandro.Genoni@univ-lorraine.fr
}

The X-ray restrained/constrained wavefunction (XRW/XCW) fitting approach $[1,2]$ is one of the well-established methods of modern quantum crystallography [3-5], an approach that allows the determination of wavefunctions from experimental X-ray diffraction measurements by minimizing a functional (i.e., the so-called Jayatilaka functional) given by the sum of the electronic energy of the system under exam and of the statistical agreement between experimental and computed structure factor amplitudes.

Initially proposed in the framework of the restricted Hartree-Fock formalism [1,2], over the years the method has been gradually extended to other techniques of quantum chemistry (e.g., unrestricted Hartree-Fock formalism, density functional theory, relativistic methods and extremely localized molecular orbital (ELMO) strategy), but practically remaining always limited to a single Slater determinant ansatz for the wavefunction to be determined.

In this presentation, two recent XRW fitting techniques that went beyond the previous limitation will be discussed: the X-ray restrained extremely localized molecular orbital - valence bond (XR-ELMO-VB) method $[6,7]$ and the X-ray restrained spin-coupled (XRSC) strategy $[8,9]$. Both of them are multi-determinant XRW approaches and, above all, they are strongly rooted in Valence Bond theory, thus allowing the extraction of traditional chemical descriptors (e.g., weights of resonance structures) compatible with measured X-ray diffraction data

The XR-ELMO-VB technique, which can be considered as the first prototype multi-determinant XRW approach, exploits the use of pre-computed and frozen ELMOs to define the basis of pre-determined Slater determinants on which expanding the desired wavefunction [6]. The method is particularly useful to investigate systems characterized by a multi-reference character. The performed calculations have also clearly shown that the technique is able to reveal how the relative importance of resonance structures changes when structure factors measured at different conditions (e.g., at ambient or high pressure) are used as external restraints [7].

The XCSC method can be considered a step forward compared to the XR-ELMO-VB strategy. In fact, through the coupling of the XRW philosophy with the spin-coupled technique of Valence Bond theory, the approach enables not only the determination of resonance structure weights, but also the optimization of the so-called spin-coupled orbitals, which are quite localized and allow us to shed light on the spatial rearrangements of the electronic clouds and the hybridization of atoms $[8,9]$. Preliminary tests have shown that XCSC computations can provide resonance structure weights, spin-coupled orbitals and global electron density distributions that are different from those obtained through corresponding gas-phase calculations [9]. These differences are probably due to the capability of the X-ray restrained spin-coupled approach in getting information contained in the experimental data employed in the computations (e.g., correlation and crystal filed effects).

[1] Jayatilaka, D. (1998). Phys. Rev. Lett. 80, 798.

[2] Jayatilaka, D. \& Grimwood, D. J. (2001). Acta Cryst. A57, 76.

[3] Genoni, A., Bučinský, L., Claiser, N., Contreras-García, J., Dittrich, B.; Dominiak, P. M., Espinosa, E., Gatti, C., Giannozzi, P., Gillet, J.-M., Jayatilaka, D., Macchi, P., Madsen, A. Ø., Massa, L. J., Matta, C. F., Merz, K. M. Jr., Nakashima, P. N. H., Ott, H., Ryde, U., Schwarz, K., Sierka, M. \& Grabowsky, S. (2018). Chem. Eur. J. 24, 10881.

[4] Grabowsky, S., Genoni, A. \& Bürgi, H.-B. (2017). Chem. Sci. 8, 4159.

[5] Genoni, A. \& Macchi, P. (2020) Crystals 10, 473.

[6] Genoni, A. (2017). Acta Cryst. A73, 312.

[7] Casati, N., Genoni, A., Meyer, B., Krawczuk, A. \& Macchi, P. (2017). Acta Cryst. B73, 584.

[8] Genoni, A., Franchini, D., Pieraccini, S. \& Sironi, M. (2018). Chem. Eur. J. 24, 15507.

[9] Genoni, A., Macetti, G., Franchini, D., Pieraccini, S. \& Sironi, M. (2019). Acta Cryst. A75, 778.

Keywords: X-ray restrained wavefunction, quantum crystallography, valence bond theory.

The French Research Agency (ANR) is gratefully acknowledged for financial support of this work through the Young Investigator Project "QuMacroRef" (Grant No. ANR-17-CE29-0005-01).

Acta Cryst. (2021), A77, C66 